\title{
Cochrane Review Summary: Mini-Mental State Examination (MMSE) for the detection of dementia in clinically unevaluated people aged 65 and over in community and primary care populations
}

\author{
Daksha Trivedi \\ First published online 5 June 2017 \\ Key words: Alzheimer's; dementia; diagnosis; older people; primary care
}

Senior Research Fellow, Centre for Research in Primary and Community Care, University of Hertfordshire, Hatfield, UK

\section{Review question}

What is the diagnostic accuracy of the Mini-Mental State Examination (MMSE) in community dwelling people aged 65 years and over?

\section{Relevance to primary care and nursing}

Primary health care professionals including specialist nurses have an integral role to play in the diagnosis and assessment of dementia and the provision of ongoing support for patients and their carers (National Institute for Health and Clinical Excellence, 2006; National Health Service England, 2014).

\section{Characteristics of the evidence}

Included studies examined the diagnostic accuracy of the MMSE in people considered to have a memory problem (by patient, informant or clinician), as well as screening studies that examined the diagnostic accuracy in people regardless of a memory complaint (asymptomatic people). They compared the 11-item (maximum score 30) MMSE test (at any cut point) in people who had not

Correspondence to: Dr Daksha Trivedi, Senior Research Fellow, Centre for Research in Primary and Community Care, University of Hertfordshire, College Lane, Hatfield AL10 9AB, UK. Email: d.trivedi@herts.ac.uk

(C) Cambridge University Press 2017 previously been evaluated versus a commonly accepted clinical reference standard for all-cause dementia (defined by Diagnostic and Statistical Manual of Mental Disorders; International Classification of Diseases, the Clinical Dementia Rating) and subtypes (Alzheimer disease dementia, Lewy body dementia, vascular dementia, frontotemporal dementia). This Cochrane review contained 48 studies (cross-sectional or nested case control) that administered the MMSE test and the reference standard within less than six months in primary care and community settings (Creavin et al., 2016). Studies spanned various countries including the United States, Europe with MMSE versions in various languages from different parts of the world.

Excluded studies were those involving people diagnosed with dementia or in secondary care, those recruited based on specific chronic conditions (eg, other neurological conditions, such as Parkinson's disease), using classic case control design (where cases are known at the outset) or with a history of substance abuse, central nervous system trauma, tumour or infection as the prevalence of dementia may differ from the general population.

\section{Summary of key evidence}

In total, 28 studies in community settings were pooled in a meta-analysis and six in primary care. 
In all, 14 community-based studies were not suitable for pooling. Studies were overall of low risk of bias (Quality assessment using QUADAS-2 checklist) although in some analysis, wide confidence intervals (CI) due to limitations of data available suggested uncertainty in the results. Participants in meta-analysis ranged from 37 to 2727 [median 314, interquartile range (IQR) 160-647].

The diagnostic accuracy was reported at 18 cut points (MMSE score 10,14-30 inclusive) in the community and 10 cut points (MMSE score 17-26 inclusive) in primary care studies. The sensitivity and specificity with $95 \%$ CI and dementia prevalence are presented for studies that were pooled for analysis.

\section{Community studies}

MMSE at cut point 24 (indicating normal)

In total, 15 studies, sensitivity 0.85 (95\% CI 0.74-0.92), specificity 0.90 (95\% CI $0.80-0.95$ ); dementia prevalence median $7.4 \%$ (IQR 5.5$20.1 \%$ ). This suggests that $85 \%$ of people with dementia are likely to be correctly identified with the MMSE, while $15 \%$ would be incorrectly classified as not having dementia or false negatives; $90 \%$ of those tested would be correctly identified as not having dementia whilst $10 \%$ would be false positives and may need to have further tests.

\section{MMSE at cut point 25}

In total, 10 studies, sensitivity 0.87 (95\% CI $0.78-0.93$ ), specificity 0.82 (95\% CI $0.65-0.92)$, dementia prevalence $8.4 \%$ (IQR 6.0-19.0\%). When adjusted for education, specificity was 0.70 (95\% CI 0.50-0.85) and sensitivity 0.97 (95\% CI 0.83-1.00), from seven studies of which two were at high risk of bias. Dementia prevalence median was $13.8 \%$ (IQR $2.4-27.4 \%$ ).

\section{Primary care studies}

Each cut point was reported by only three studies, and a summary of sensitivity and specificity was not feasible due to lack of adequate data and considerable heterogeneity. Due to insufficient data, analysis of diagnostic accuracy for dementia subtypes was not possible.

Primary Health Care Research \& Development 2017; 18: 527-528

\section{Implications for practice}

MMSE can be used by clinicians and patients as part of the process for evaluating the diagnosis of dementia but results should be considered carefully both in the clinical context as well as the wider context of the individual patient's characteristics such as personality, behaviour and their ability to manage the condition at home.

\section{Implications for research}

Future research needs to consider the pathway of diagnosis experienced by the patient and to examine the diagnostic accuracy of MMSE in this context to inform how MMSE tests (from the perspective of patients, carers and clinicians) impact on patient relevant outcomes.

\section{Acknowledgements}

The author is a member of the Cochrane Nursing Care Field (CNCF).

\section{Financial Support}

This research received no specific grant from any funding agency, commercial or not-for-profit sectors.

\section{Conflicts of Interest}

None.

\section{References}

Creavin, S.T., Wisniewski, S., Noel-Storr, A.H., Trevelyan, C.M., Hampton, T., Rayment, D., Thom, V.M., Nash, K.J.E., Elhamoui, H., Milligan, R., Patel, A.S., Tsivos, D.V., Wing, T., Phillips, E., Kellman, S.M., Shackleton, H.L., Singleton, G.F., Neale, B.E., Watton, M.E. and Cullum, S. 2016: Mini-Mental State Examination (MMSE) for the detection of dementia in clinically unevaluated people aged 65 and over in community and primary care populations. Cochrane Database of Systematic Reviews, Issue 1. Art. No. CD011145. https://doi.org/10.1002/14651858.CD011145.pub2

National Institute for Health and Clinical Excellence. 2006: Dementia: supporting people with dementia and their carers in health and social care. Clinical Guideline NICE. Retrieved 6 February 2017 from org.uk/guidance/cg42

National Health Service England. 2014: Best practice in memory services: learning from across England. Retrieved 6 February 2017 from https://www.england.nhs.uk/wp-content/uploads/2014/12/memory-clinics-final.pdf 